\title{
Research on Supplier Selection of Epc Project Based on Fuzzy Topsis
}

\author{
Xiaoting Yang ${ }^{1}$, Shixia Duan ${ }^{1}$ \\ ${ }^{1}$ Management and engineering Department, Zhengzhou University, Henan and 450001, China \\ 710613286@qq.com,469900455@qq.com
}

Keywords: EPC project; Supplier selection; Fuzzy TOPSIS.

\begin{abstract}
The EPC mode has gradually become the mainstream in the field of engineering construction in China, which is clearly stated in the"13th Five-Year Plan" issued by the State Council. Procurement as EPC general contract in the economy accounted for an important part of the larger, shortened the delivery time, reduceed the overall procurement costs, so as to win more profit margins for enterprises. Therefore, this paper, from the perspective of general contracting, combines the EPC resource integration characteristics and the supply chain management theory, constructs the fuzzy entropy TOPSIS selection model and the specific decision step. Finally, the specific application of this method in bidding is analyzed with an actual case, so as to provide a reference for the supplier selection decision of
\end{abstract} the EPC general contracting project.

\section{INTRODUCTION}

In recent years, EPC general contracting (Engineering-Procurement-Construction) has been widely used in China because of its advantages of efficient resource integration. The mode is mainly based on the design as a leader, the procurement link, based on the construction to achieve the final function of the project. EPC can effectively optimize the project management, access to the construction enterprises, the market and the government's greater recognition. And material procurement links forward convergence design, backward connection construction, it is an important intermediate bridge. For the EPC general contracting project, the procurement stage accounts for $50-70 \%$ of the contract fee. The Japanese scholar Mr. Nishizawa (Jun Zhu,2016) put forward that procurement is the "third largest profit source", so the core of reducing the project cost is high quality and price economy of raw materials. EPC general contractor procurement on the one hand requires the price and quality of the economy, on the other hand requires timely delivery to cooperate with the construction, and this established goal only through reliable suppliers to provide protection for enterprises. Therefore, this paper from the perspective of the general contractor to cost control as a foothold, combined with supply chain management theory, research EPC project supplier selection problem.

\section{EPC PROJECT PROCUREMENT AND SUPPLIER MANAGEMENT}

\subsection{Under EPC mode procurement management}

Supply chain management theory originated in the field of manufacturing, has gradually begun to introduce the field of engineering construction, in order to solve the construction of procurement management problems encountered. For the general contractor, the EPC mode has the advantage of integrating resources and information from all sides, using supply chain management theory to guide procurement management, helping to improve procurement performance and cost optimization. EPC mode of procurement management both the general contract model of the common procurement, but also its own characteristics: the strong professional of procurement, the large amount of money occupied, the procurement cycle is tense, the poor versatility of equipment, the owners of the procurement requirements more stringent, etc.(Hao Zhang,2015). With the development of BIM technology, EPC contractors use modular design to control project costs, which makes the original part of the assembly and commissioning part of the conversion to the procurement part of the completion of this change more emphasis on procurement management on the project cost of the important role.

In the EPC project, the procurement department should not only design documents and related standards as a guide, but also to ensure the provision of high quality qualified products to the construction sector to ensure timely construction. The main contents of the procurement work include: a large number of raw materials, related logistics and warehousing, equipment supplier management, index optimization, procurement convergence, etc (Guangming Ren,2015). In the procurement management of the general contractor not only concerned about the material, equipment prices and supplier chain, while using their own integrated advantages of integration of information systems resources. On the one hand the use of supply chain as a whole perspective, in the procurement of links between the rapid transfer of information, both to ensure quality and efficiency, but also cost savings; the other hand, the general contractor and supply chain links to establish cooperation relationship, both to achieve long-term cooperation, but also to ensure the effective link between the various links. The EPC project can ultimately improve the cost performance, quality performance, schedule performance and warehouse performance evaluation in purchasing management through supply chain management (Wenzhe Tang et al,2017). 


\subsection{Under EPC mode supplier selection}

Excellent supplier is the guarantee of the project function and the effective source of the profit of the contractor. After the purchase contract is signed, the purchase cost of the whole project is basically determined. The choice of supplier can play the role of optimizing the cost of the entire supply chain. In the EPC mode, the project's life cycle are considering long-term reduction in procurement costs, so the supplier's choice more and more attention by the general contractor.

First of all, EPC project to select supplier should actively implement the bidding mechanism. Bidding mechanism can be done to expand the scope of choice of supplier resources, you can choose a wide range of reputable, comprehensive strength of the enterprise. At the same time EPC project equipment is large and complex high-specific equipment, public bidding method can filter the supplier in the technical standard, you can also do multi-index evaluation to select supplier.

Secondly, evaluation index system should be established to strengthen the supplier selection and evaluation management. In the EPC mode, the evaluation index system should be established according to the actual project. The supplier's selection evaluation should include the product quality, the price, the purchasing cycle and the after-sales service level. The supplier's performance evaluation should also include the quality target, the cost target, the progress target , logistics objectives and other aspects of the completion of the situation (Ting He et al,2014). Common evaluation methods are: analytic hierarchy process, fuzzy comprehensive evaluation method, entropy method, TOPSIS(Technique for Order Preference by Similarity to an Ideal Solution) method, data envelopment analysis, gray correlation analysis(Yanfang Huo and Bowen $\mathrm{Wu}$,2015). The general contractor can not only select the reasonable supplier through the evaluation index, but also according to the supplier's performance performance score, the supplier to take incentive measures to ensure that suppliers improve their products and services.

Thirdly, supplier strategic cooperative partnership should be established. EPC contractors because of its integrated advantages in the procurement, should establish their own long-term cooperation, mutual trust in the strategic partnership(Suicheng Li et al,2016).The establishment of a strategic partnership can establish a reciprocal relationship between the general contractor and the supplier, while at the same time selecting the supplier and giving the supplier a sense of belonging to the general contractor, and there is a competitive relationship between the supplier and itself. The relationship between the network, in the strategic cooperation system has been unified and sublimation, the formation of a collective consciousness, more conducive to achieving the project objectives.

\section{RESEARCH METHOD AND EVALUATION MODEL}

Bid evaluation is an important part of the tender procurement, in accordance with the evaluation of the order to determine the supplier, the cost of the project procurement stage has been basically determined. So the scientific and economic evaluation of the project directly affect the profit margins. The minimum bid method is generally applicable to projects with general technical and performance standards, while EPC projects are mostly large and complex projects, it involves the procurement of equipment and materials for high. It can not simply to the lowest bid method to choose a supplier, this approach may not only reduce the cost of the project, but also bring a series of other losses. Therefore, supplier selection is very important in the procurement. Meanwhile the evaluation process of its scientific evaluation by the expert experience and subjective judgments affect the larger. The TOPSIS method is used to sort the limited evaluation object and the ideal solution and it has obvious advantages in solving multiple attribute decision making problems. It has high flexibility, for the project to provide a reliable basis for bidding. In the TOPSIS method, the fuzzy number theory is introduced so that the qualitative index can be quantified reliably, and the intuitive fuzzy entropy method can allocate the weight of the evaluation index scientifically and rationally (Shanliang Yang et al,2015).

\subsection{Establishment of evaluation index system}

Table 1: EPC project supplier selection evaluation index system.

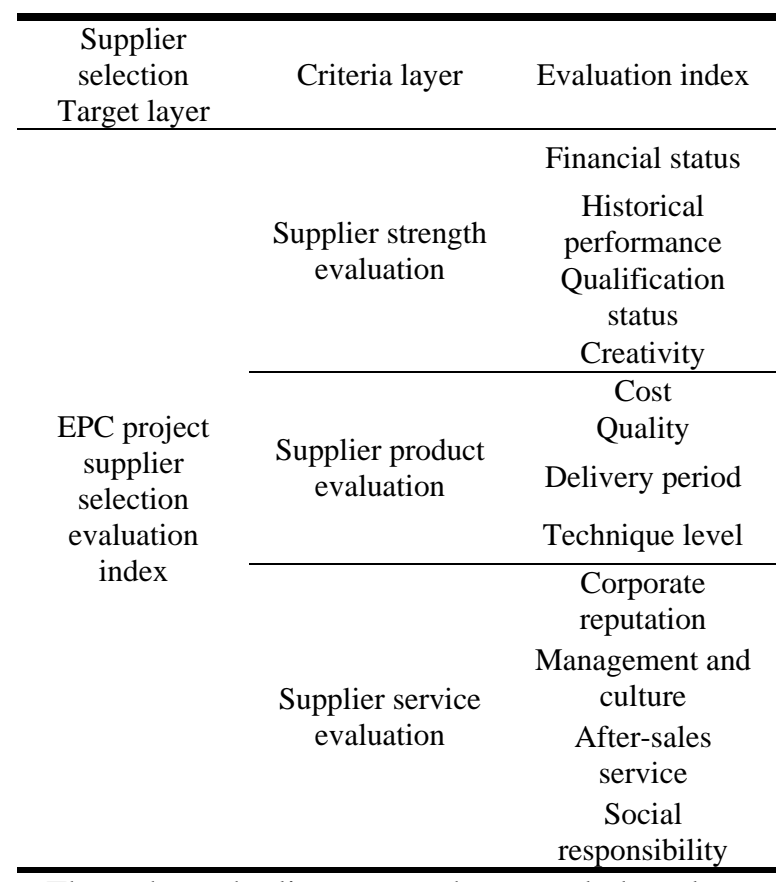

Throughout the literature at home and abroad, many scholars have contributed to the study of supplier evaluation index, but there are few researches on EPC mode evaluation index system. Based on analysis of the existing literature and the practical characteristics of EPC project, this paper build a set of practical supplier selection and evaluation indexes based on the basic principles of scientific, systematic, hierarchical, comparability and dynamic system (Xiao Zhang,2014), as shown in Table 1.

\subsection{Define the evaluation set}

In the constructed evaluation model, we assume that : 
$\mathrm{S}=\{\mathrm{S} 1, \mathrm{~S} 2 \ldots \mathrm{Sm}\}$ is the set of tender suppliers; $E=\{E 1, E 2 \ldots E n\}$ is the set of evaluation index; $\mathrm{D}=\{\mathrm{D} 1, \mathrm{D} 2 \ldots \mathrm{Dk}\}$ is the set of decision makers; $=\{1,2, \ldots n\}$ is the weight set of the evaluation index; is the evaluation matrix. And is the evaluation value of the i-th supplier in the index $\mathrm{Cj}$; indicates that the k-th decision maker uses the natural language variable to evaluate the value of the $i$-th supplier in the $j$ th index. It is assumed that the weight of each expert involved in the evaluation is the same, while $\mathrm{j}$ is completely unknown and satisfies a value greater than or equal to 0 , whose sum equals 1 . The weight of the index and the evaluation matrix are quantified by the decision maker in the form of natural language variables and converted into corresponding fuzzy numbers. In this paper, the language evaluation information into intuitive fuzzy number (Zhen Zhang and Chonghui Guo,2011). The variable tag and the corresponding fuzzy number is shown in Table 2.

Table 2: This caption has more than one line so it has to be set to justify.

\begin{tabular}{lll}
\hline $\begin{array}{c}\text { Weight } \\
\text { Language variable }\end{array}$ & \multicolumn{1}{c}{$\begin{array}{c}\text { Evaluation } \\
\text { Language variable }\end{array}$} & $\begin{array}{c}\text { Intuitive fuzzy } \\
\text { number }\end{array}$ \\
\hline EL (Extreme Little) & EB (Extreme Bad) & $(0.05,0.95,0.00)$ \\
VL (Very Little) & VB (Very Bad) & $(0.15,0.80,0.05)$ \\
L (Little) & B (Bad) & $(0.25,0.65,0.10)$ \\
ML (Medium Little) & MB (Medium Bad) & $(0.35,0.55,0.10)$ \\
M (Medium) & M (Medium) & $(0.50,0.40,0.10)$ \\
MH (Medium & MG (Medium & $(0.65,0.25,0.10)$ \\
Heavy) & Good) & $(0.75,0.15,0.10)$ \\
H (Heavy) & G (GOOD) & $(0.85,0.10,0.05)$ \\
VH (Very Heavy) & VG (Very Good) & $(0.95,0.05,0.00)$ \\
\hline EH (Extreme & EG (Extreme Good) & \\
\hline
\end{tabular}

\subsection{Specific decision steps}

Step1: Collect the results of the natural language variables that given by each decision maker, including the evaluation of the weight of the index and the evaluation matrix.

Step2: The language variable is transformed into fuzzy number, and the weight of evaluation index is determined by intuitive fuzzy entropy method.

Assuming that the evaluation index is the fuzzy number (a, b, c), define the intuitive fuzzy entropy as(Mingmei Gao et al,2014; Qinghua Zhou and Qi Wang,2017):

$$
H_{j}=\frac{1}{m} \sum_{i=1}^{m} \frac{1-|a-b|^{2}+c^{2}}{2}
$$

The entropy of each evaluation index is:

$$
\omega_{j}=\frac{1-H_{j}}{n-\sum_{j=1}^{n} H_{j}}, \forall i, j
$$

Step3: The language variables are transformed into fuzzy numbers, and the expert evaluation matrix is quantified with fuzzy numbers.

$$
X_{i j}=\frac{1}{k} \otimes \sum_{k=1}^{k} X(k)_{i j}
$$

Step4: The evaluation matrix is normalized to obtain a standardized fuzzy matrix Y.

$$
Y_{i j}=\left(\frac{X_{i j 1}}{\max \left(X_{i j 1}\right)}, \frac{X_{i j 2}}{\max \left(X_{i j 2}\right)}, \frac{X_{i j 3}}{\max \left(X_{i j 3}\right)}\right)
$$
Z.

Step5: Calculate the weighted decision fuzzy matrix

$$
Z_{i j}=Y_{i j} \otimes \omega_{j}=\left(Y_{i j 1} \otimes \omega_{j 1}, Y_{i j 2} \otimes \omega_{j 2}, Y_{i j 3} \otimes \omega_{j 3}\right)
$$

Step6: Determine the fuzzy positive and negative ideal solutions and.

$$
\begin{aligned}
& Z^{+}=\left(Z_{1}^{+}, Z_{2}{ }^{+}, \cdots, Z_{\mathrm{j}}^{+}, \cdots, Z_{\mathrm{n}}^{+}\right), \\
& Z_{\mathrm{j}}^{+}=\max _{i} Z_{i j}, \forall \mathrm{j} \\
& Z^{-}=\left(Z_{1}^{-}, Z_{2}^{-}, \cdots, Z_{\mathrm{j}}^{-}, \cdots, Z_{\mathrm{n}}^{-}\right), \\
& Z_{\mathrm{j}}^{-}=\min _{i} Z_{i j}, \quad \forall \mathrm{j}
\end{aligned}
$$

Step7: Calculate the distance between each supplier and the fuzzy positive ideal solution and the negative ideal solution.

$$
\begin{aligned}
& D_{i}^{+}=\sum_{j=1}^{n} D\left(Z_{i j}, Z_{j}^{+}\right), \forall i, j \\
& D_{i}^{-}=\sum_{j=1}^{n} D\left(Z_{i j}, Z_{j}^{-}\right), \forall i, j
\end{aligned}
$$

Where the two fuzzy numbers are and, then the distance between them is(CHEN S M,2001):

$$
D(a, b)=\sqrt{\frac{1}{3}\left[\left(a_{1}-b_{1}\right)^{2}+\left(a_{2}-b_{2}\right)^{2}+\left(a_{3}-b_{3}\right)^{2}\right]}
$$

Step8: Calculate the proximity of each supplier to the ideal solution and sort it.

$$
C_{i}=\frac{D_{i}^{-}}{D_{i}^{+}+D_{i}^{-}}, \forall i
$$

The larger the value of , the closer the supplier is to the positive ideal solution, the farther away from the negative ideal solution, the more forward the supplier's ranking. So it can be sorted according to the value of .

\section{CASE ANALYSIS}


An EPC general contractor want to purchase a largescale mechanical and electrical equipment, and adopt external public bidding procurement. There were four suppliers(S1,S2,S3,S4) to participate in the tender. In order to make the evaluation process more scientific and reasonable to optimize the procurement costs, the EPC contractor has composed of five expert review team (D1, D2, D3,D4,D5)to evaluate. According to the existing theoretical basis and literature achievements, and according to the evaluation index system established in Table 1, the evaluation indexes of the procurement are as follow: Quality E1,Cost E2, Delivery Period E3, Technical Level E4, Quality of Service E5, Corporate Reputation E6, Similar Project Performance E7, Innovation Ability E8. Among them Cost and Delivery
Period are quantitative indicators, so the smaller the value in the tender documents, the higher the expert evaluation (Chengwei Zhao et al,2016). S1 bid offer is 415 million, the delivery period is 80 days; S2 bid offer is 385 million, the delivery period is 70 days; S3 bid offer is 400 million, the delivery period is 60 days; S4 bid offer is 440 million, the delivery period is 65 days.

According to the above evaluation methods and implementation steps, the expert evaluation team's fuzzy evaluation of the weight of the evaluation index and the fuzzy evaluation of the various indexes of the suppliers, the standardized fuzzy matrix and the weighted decision fuzzy matrix(Jian Chen et al,2016) are shown in Table 3,Table 4,Table 5 and Table 6.

Table 3: Fuzzy evaluation of experts' weight of evaluation index.

\begin{tabular}{ccccccccc}
\hline $\begin{array}{c}\text { Decision } \\
\text { makers }\end{array}$ & E1 & E2 & E3 & E4 & E5 & E6 & E7 & E8 \\
\hline D1 & EH & EH & VH & VH & VH & MH & MH & H \\
D2 & EH & EH & EH & VH & H & VH & H & MH \\
D3 & H & EH & VH & EH & MH & H & VH & VH \\
D4 & EH & VH & EH & VH & MH & VH & H & MH \\
D5 & VH & EH & H & EH & H & VH & VH & M \\
$\omega j$ & 0.138 & 0.1456 & 0.1338 & 0.1373 & 0.1085 & 0.1188 & 0.1153 & 0.1027 \\
\hline
\end{tabular}

Table 4: Experts on the various indicators of the fuzzy evaluation of supplier.

\begin{tabular}{|c|c|c|c|c|c|c|c|c|c|}
\hline $\mathrm{S}$ & D & E1 & E2 & E3 & E4 & E5 & E6 & E7 & E8 \\
\hline \multirow{5}{*}{ S1 } & D1 & $\mathrm{G}$ & $\mathrm{M}$ & $\mathrm{M}$ & G & G & VG & VG & MG \\
\hline & D2 & VG & MB & B & MG & G & G & MG & G \\
\hline & D3 & EG & MB & MB & G & MG & MG & VG & $\mathrm{M}$ \\
\hline & D4 & VG & $\mathrm{M}$ & MB & VG & MG & G & $\mathrm{G}$ & G \\
\hline & D5 & VG & MB & $\mathrm{M}$ & G & G & MG & G & MG \\
\hline \multirow{5}{*}{ S2 } & D1 & MG & VG & MG & $\mathrm{G}$ & MG & $\mathrm{G}$ & MG & G \\
\hline & D2 & MG & EG & G & VG & $\mathrm{G}$ & MG & $\mathrm{G}$ & MG \\
\hline & D3 & $\mathrm{M}$ & VG & $\mathrm{M}$ & G & G & VG & G & G \\
\hline & D4 & G & VG & MG & G & MG & G & G & VG \\
\hline & D5 & $\mathrm{M}$ & EG & MB & VG & VG & G & MG & G \\
\hline \multirow{6}{*}{ S3 } & D1 & G & $\mathrm{G}$ & VG & $\mathrm{M}$ & VG & $\mathrm{G}$ & MG & $\mathrm{G}$ \\
\hline & D2 & MB & $\mathrm{M}$ & G & $\mathrm{M}$ & G & MG & $\mathrm{M}$ & G \\
\hline & D3 & $\mathrm{M}$ & MG & VG & MB & G & $\mathrm{M}$ & G & MG \\
\hline & D4 & $\mathrm{M}$ & MG & G & MB & VG & $\mathrm{M}$ & MB & $\mathrm{G}$ \\
\hline & D5 & MG & $\mathrm{G}$ & VG & B & G & MG & $\mathrm{M}$ & MG \\
\hline & Xij & $(0.55,0.35,0.10)$ & $(0.66,0.24,0.10)$ & $(0.81,0.12,0.07)$ & $(0.39,0.51,0.10)$ & $(0.79,0.13,0.08)$ & $(0.61,0.29,0.10)$ & $(0.55,0.35,0.10)$ & $(0.71,0.19,0.10)$ \\
\hline \multirow{6}{*}{ S4 } & D1 & VG & B & VG & MG & G & G & MG & VG \\
\hline & D2 & G & MB & MG & G & MG & G & MG & G \\
\hline & D3 & VG & EB & G & G & G & MG & G & VG \\
\hline & D4 & VG & VB & MG & VG & M & G & VG & MG \\
\hline & D5 & G & B & G & G & MG & MG & G & G \\
\hline & Xij & $(0.81,0.12,0.07)$ & $(0.21,0.72,0.07)$ & $(0.73,0.18,0.09)$ & $(0.75,0.16,0.09)$ & $(0.66,0.24,0.10)$ & $(0.71,0.19,0.10)$ & $(0.73,0.18,0.09)$ & $(0.77,0.15,0.08)$ \\
\hline
\end{tabular}


Table 5: Standardized fuzzy matrix.

\begin{tabular}{|c|c|c|c|c|c|c|c|c|}
\hline S & E1 & E2 & E3 & E4 & E5 & E6 & E7 & E8 \\
\hline S1 & $(1,0.286,0.5)$ & $(0.461,0.625,1)$ & $(0.481,1,1)$ & $(0.949,0.314,0.9)$ & $(0.899,0.792,1)$ & $(0.973,0.621,0.9)$ & $(1,0.429,0.8)$ & $(0.857,1,1)$ \\
\hline S2 & $(0.718,0.829,1)$ & $(1,0.111,0.3)$ & $(0.716,0.627,1)$ & $(1,0.255,0.8)$ & $(0.924,0.75,0.9)$ & $(1,0.552,0.9)$ & $(0.922,0.543,1)$ & $(0.974,0.667,0.9)$ \\
\hline S3 & $(0.647,1,1)$ & $(0.742,0.333,1)$ & $(1,0.235,0.7)$ & $(0.494,1,1)$ & $(1,0.542,0.8)$ & $(0.813,1,1)$ & $(0.714,1,1)$ & $(0.922,0.792,1)$ \\
\hline S4 & $(0.953,0.343,0.7)$ & $(0.236,1,0.7)$ & $(0.901,0.353,0.9)$ & $(0.949,0.314,0.9)$ & $(0.835,1,1)$ & $(0.947,0.655,1)$ & $(0.948,0.514,0.9)$ & $(1,0.625,0.8)$ \\
\hline
\end{tabular}

Table 6: Weighted decision fuzzy matrix.

\begin{tabular}{|c|c|c|c|c|c|c|c|c|}
\hline$S$ & E1 & E2 & E3 & E4 & E5 & E6 & E7 & E8 \\
\hline S1 & $\begin{array}{c}(0.138,0.039 \\
0.069)\end{array}$ & $\begin{array}{c}(0.067,0.091 \\
0.146)\end{array}$ & $\begin{array}{c}(0.064,0.134 \\
0.134)\end{array}$ & $\begin{array}{c}(0.13,0.043 \\
0.124)\end{array}$ & $\begin{array}{c}(0.098,0.086 \\
0.109)\end{array}$ & $\begin{array}{c}(0.116,0.074 \\
0.107)\end{array}$ & $\begin{array}{c}(0.115,0.049 \\
0.092)\end{array}$ & $\begin{array}{c}(0.088,0.103 \\
0.103)\end{array}$ \\
\hline S2 & $\begin{array}{c}(0.099,0.114 \\
0.138)\end{array}$ & $\begin{array}{c}(0.146,0.016 \\
0.044)\end{array}$ & $\begin{array}{c}(0.096,0.083 \\
0.134)\end{array}$ & $\begin{array}{c}(0.137,0.035 \\
0.11)\end{array}$ & $\begin{array}{c}(0.1,0.081 \\
0.098)\end{array}$ & $\begin{array}{c}(0.119,0.066 \\
0.107)\end{array}$ & $\begin{array}{c}(0.106,0.063 \\
0.115)\end{array}$ & $\begin{array}{c}(0.1,0.069 \\
0.092)\end{array}$ \\
\hline S3 & $\begin{array}{c}(0.089,0.138 \\
0.138)\end{array}$ & $\begin{array}{c}(0.108,0.048 \\
0.146)\end{array}$ & $\begin{array}{c}(0.134,0.031 \\
0.094)\end{array}$ & $\begin{array}{c}(0.068,0.137 \\
0.137)\end{array}$ & $\begin{array}{c}(0.109,0.059 \\
0.087)\end{array}$ & $\begin{array}{c}(0.097,0.119 \\
0.119)\end{array}$ & $\begin{array}{c}(0.082,0.115 \\
0.115)\end{array}$ & $\begin{array}{c}(0.095,0.08 \\
0.103)\end{array}$ \\
\hline
\end{tabular}

According to the formula (6) and (7), the fuzzy positive ideal solution and the negative ideal solution of the procurement tender are calculated respectively:

$$
Z^{+}=\{(0.138,0.039,0.069),(0.146,0.016,0.044),(0.13
$$
4,0.031,0.094),0.137,0.035,0.11),(0.109,0.059,0.087), (0. 119,0.066,0.107),(0.115,0.049,0.092), , (0.103,0.064,0.08 2) \};

$$
Z^{-}=\{(0.089,0.138,0.138),(0.034,0.146,0.102),(0.064
$$

,0.134,0.134),(0.068,0.137,0.137),(0.091,0.109,0.109),(0

Table 7: The proximity of each supplier to the ideal solution.

\begin{tabular}{ccccc}
\hline Suppliers & Di+ & Di- & Ci & Rank \\
\hline S1 & 0.3575 & 0.442 & 0.553 & 3 \\
S2 & 0.2374 & 0.5246 & 0.688 & 1 \\
S3 & 0.5015 & 0.3112 & 0.383 & 4 \\
S4 & 0.3227 & 0.4447 & 0.579 & 2 \\
\hline
\end{tabular}

From the final sorting results, we can know that the preferred order is: the supplier $2>$ the supplier $4>$ the supplier $1>$ the supplier 3 , so the supplier 2 is recommended as the final winner.

\section{CONCLUSIONS}

Based on the fuzzy influence of purchasing TOPSIS, this paper proposes a multi-index supplier selection decision model. This model has the advantages of wide application, intuitive and low distortion. Through this model, EPC general contractor can be supplier selection to implement a scientific economy and reasonable management, so as to achieve the project objectives at the same time to further improve the profitability of enterprises. This model can be used not only for supplier comparison, but also for quantitative analysis of supplier performance evaluation. This paper provides a scientific basis for the EPC general contractor to select suppliers. But the factors that affect the interaction between risk factors and evaluation indicators are not considered in the study, so further research is needed to achieve the project cost target so that the enterprise can get higher of
$.097,0.119,0.119),(0.082,0.115,0.115),(0.088,0.103,0.10$ 3)\}.

According to the formula (8) - (10) to calculate the bidding scheme to the positive and negative ideal solution of the distance, and then according to formula (11) to obtain the program close degree and sort, as shown in Table 7.

the profit margins.

\section{REFERENCES}

[1] CHEN S M, 2001. Fuzzy group decision making for evaluating the rate of aggregative risk in software development, Fuzzy Sets and Systems. 118(1), pp. 87-89.

[2] Chengwei Zhao, Xiongbao Dong and Zhenglong Zhou, 2016. Application of TOPSIS method based on fuzzy theory in engineering project evaluation, Practice and understanding of mathematics.46(8), pp. 99-105.

[3] Guangming Ren, 2015. International project to improve EPC procurement management model under the integration of supply chain, Heilongjiang science. (10), pp. 104-105.

[4] Hao Zhang,2015. Procurement management under the international EPC contract mode, International economic cooperation. (3), pp. 89-95.

[5] Jian Chen, Shengliang Zhang and Xin Li, 2016. Research on relative green degree of environmental awareness design scheme based on fuzzy AHP-TOPSIS, Science and technology review. 34(18), pp. 304-313.

[6] Jun Zhu, 2016. Research on purchasing cost control of industrial project under EPC mode, Construction supervision. (11), pp. 24-27.

[7] Mingmei Gao, Tao Sun and Jianjun Zhu, 2014. An 
improved intuitionistic fuzzy entropy axiomatic definition and construction formula, Control and decision. (3), pp. 470-474.

[8] Qinghua Zhou, Qi Wang,2017. Based on intuitionistic fuzzy set VIKOR's low carbon supplier selection method, Technology management research. 37(10), pp. 233-237.

[9] Shanliang Yang, Peng Wang and Ge Li, 2015. Based on the intuitive fuzzy VIKOR method, the decision model of optimization group is proposed, System simulation journal. 27(9), pp. 2169-2175.

[10] Suicheng Li, Mengchao $\mathrm{Wu}$ and $\mathrm{Na} \mathrm{Li}, 2016$. Study on the influence mechanism of strategic supplier management on supplier network integration, Nankai management review. 19(16), pp. 58-69.

[11] Ting He, Weidan Zhou and Zhifang He,2014. Supplier selection research under supply chain risk management, Journal of southeast university (philosophical social science edition). (s1), pp. 88-93.

[12] Wenzhe Tang, Zhen Lei and Shuli Wang,2017. International engineering EPC project procurement integration management, Journal of tsinghua university (natural science edition). (8), pp. 838-844.

[13] Xiao Zhang,2014. Research on supplier selection of pharmaceutical enterprises based on AHP, Journal of southeast university (philosophical social science edition). (s1), pp. 31-34. [14] Yanfang Huo, Bowen Wu,2015. Supplier management inventory partner selection in fuzzy environment, Technology management research. (5), pp. 199-204.

[15] Zhen Zhang, Chonghui Guo,2011. A multi-attribute group decision method based on binary semantic information processing, Control and decision. 26(12), pp. 1881-1885. 